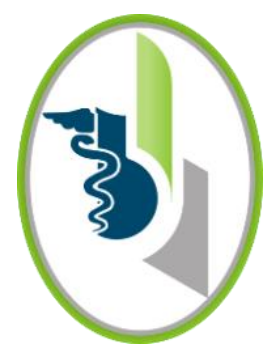

ACCESO abierto
Para citaciones: Correa, O., Camacho, A. (2020). Consulta pediátrica prenatal: objetivos y beneficios. Revista Ciencias Biomédicas, 9(2), 151-155.

Recibido: 18 de abril de 2020 Aprobado: 3 de junio de 2020

Autor de correspondencia: Oscar Correa Jiménez olcorreaj@unal.edu.co

Editor: Inés Benedetti. Universidad de Cartagena-Colombia.

\section{Consulta pediátrica prenatal: objetivos y beneficios}

\author{
Pediatric prenatal clinic: objectives and benefits
}

Oscar Correa-Jiménez ${ }^{1}$ iD, Ángela Camacho-Lindo ${ }^{2}$
${ }^{1}$ División de Inmunología Clínica. HOMI Fundación Hospital Pediátrico la Misericordia, Bogotá, Colombia.
${ }^{2}$ Departamento de Pediatría. Facultad de Medicina, Universidad Nacional de Colombia, Bogotá, Colombia.

\section{RESUMEN}

En los últimos años, la pediatría ha volcado su atención del niño en forma individual a la atención en salud este en el contexto familiar. Debido a sus beneficios, la Academia Americana de Pediatría ha recomendado la consulta pediátrica prenatal, la cual no es objeto de práctica común en nuestro medio. Este artículo busca contextualizar en qué consiste este tipo de consulta, así como sus objetivos y beneficios. La consulta pediátrica prenatal consiste en una serie de acercamientos e interacciones entre los futuros padres y el pediatra, con relación a interrogantes relacionados con el futuro hijo. Sus objetivos son: establecer una relación positiva pediatra-familia, recopilar información familiar, orientar y educar sobre habilidades de crianza e identificar los aspectos de alto riesgo. En nuestro país no existen datos publicados sobre la atención pediátrica prenatal, se sabe que se realiza principalmente en la práctica pediátrica particular y en algunos servicios de medicina prepagada, pero es necesario desarrollar un conjunto de recomendaciones para llevarla a cabo.

Palabras clave: Pediatría; Diagnóstico Prenatal; Cuidado del Niño.

\section{ABSTRACT}

Recently, pediatrics has turned its attention to the individual child to health care in the family context. Due to its benefits, the American Academy of Pediatrics has recommended prenatal pediatric clinic, which is not the object of common practice in our environment. The objective of this article is to contextualize what this type of consultation consists of, as well as its objectives and benefits. The prenatal pediatric clinic consists of a series of approaches and interactions between the future parents and the pediatrician, in relation to questions related to the future child. Its objectives are: to establish a positive pediatrician-family relationship, collect family information, guide and educate on parenting skills, and identify high-risk aspects. In our country there are no published data on prenatal pediatric care, it is known that it is performed mainly in private pediatric practice and in some prepaid medicine services, but it is necessary to develop a set of recommendations to carry it out.

Keywords: Pediatrics; Prenatal Diagnosis; Child Care. 


\section{INTRODUCCIÓN}

En los últimos años, la pediatría ha volcado su foco de atención del niño en forma individual a la atención en salud este en el contexto familiar (1). En este orden de ideas, entre más temprano se dé la interacción pediatra-padres se verá afectada positivamente la atención en salud del niño. De otro lado, el pediatra en conjunto con el obstetra debe estar involucrados en la orientación a los padres para la realización de una elección informada sobre los cuidados de su futuro hijo (2). Debido a los beneficios que le han sido demostrados, y que en esta revisión desglosaremos, la Academia Americana de Pediatría desde 1996(3) ha recomendado la consulta pediátrica prenatal. Dado que, en nuestro medio, como en otros países de Latinoamérica (4), la consulta pediátrica prenatal no es objeto de práctica común, se realiza el presente artículo de reflexión con el fin de contextualizar en qué consiste este tipo de consulta, así como sus objetivos y beneficios.

\section{¿En qué consiste?}

Si bien no se encuentra una definición precisa respecto a qué es la consulta pediátrica prenatal, podríamos decir que consiste en una serie de acercamientos e interacciones entre los futuros padres y el pediatra, con relación a preocupaciones e interrogantes relacionados con el futuro hijo.

Acorde con el escenario de un país desarrollado como Estados Unidos, el contacto prenatal entre padres y pediatra inicia por vía telefónica indagando respecto a la posibilidad de atención, durante este contacto, los padres deben ser invitados a programar la consulta y preferiblemente deben ser invitados ambos a participar de ella en la medida de las posibilidades (5). De hecho, Moniz et al. mediante una encuesta con representatividad nacional en Estados Unidos identificaron que la mayoría de los padres están interesados en recibir apoyo y educación sobre pautas de crianza y cuidados del recién nacido en consultas de atención perinatal, resaltando que plantearon preferencia sobre recibir este tipo de atención antes del nacimiento de su hijo (6). Una vez realizado el primer acercamiento, se llevará a cabo la visita al consultorio pediátrico por parte de la pareja embarazada, en donde los futuros padres tendrán la oportunidad de expresar sus necesidades, intereses y preocupaciones respecto al desarrollo y la salud de su hijo, así como recibir orientación anticipada respecto a estos tópicos (5).

Desde el Comité de aspectos sicosociales de la salud del niño y la familia de la Academia Americana de Pediatría, se ha desarrollado la siguiente categorización de la consulta pediátrica prenatal (7):

1. La consulta prenatal completa: corresponde a la forma óptima de consulta, es una visita regular al consultorio con ambos padres presentes. El objetivo de esta es lograr los objetivos propuestos de la consulta prenatal (que se desarrollan en la siguiente sección del texto). En esta suele darse el establecimiento de un compromiso mutuo y de una relación profesional sólida y gratificante. Se realizan acuerdos en diversos temas como ejemplo, los horarios de atención, los honorarios, las afiliaciones laborales del pediatra, la cobertura de atención nocturna y fines de semana y la atención de emergencia, entre otros.

2. Consulta breve para conocer: primordialmente con la madre embarazada. De duración usualmente corta, en la que se sugiere enseñar a la madre las instalaciones y el personal relacionado con la atención. Recomendable para padres que aún se encuentran en la búsqueda de pediatra.

3. Contacto básico o telefónico: corresponde al primer acercamiento. Es importante recoger los datos de identificación y proporcionar de manera amigable información introductoria respecto a la atención (folletos informativos u otros formatos).

4. Consulta prenatal grupal: fomenta el apoyo mutuo entre las parejas embarazadas al tiempo que proporciona un foro de información similar a sesiones individuales tradicionales. Tiene la ventaja adicional de ahorrar el tiempo y el gasto médico.

5. No contacto: de no llevarse a cabo la orientación a los padres respecto a cuidados y salud del niño en la etapa prenatal, la misma debe iniciarse desde la primera consulta de atención neonatal. 


\section{Objetivos}

La Academia Americana de Pediatría de los Estados Unidos reconoce como objetivos de la consulta pediátrica prenatal los siguientes (5):

- Establecimiento de una relación positiva pediatra-familia.

- Recopilación de información familiar.

- Orientación y educación sobre habilidades de crianza.

- Identificación y aproximaciones a los aspectos de alto riesgo.

De otro lado, encontramos los objetivos propuestos por el Grupo PrevInfad/PAPPS Infancia y Adolescencia de la Asociación Española de Pediatría de Atención Primaria (1):

1. Iniciar la relación padres-pediatra.

2. Informar sobre lo que necesita un niño recién nacido y describir ciertas habilidades que se deben adquirir.

3. Informar sobre lactancia materna y sobre lo que suele ocurrir en un parto.

4. Dar consejos sobre estilo de vida favorable.

5. Comprobar que la gestación se está controlando de forma adecuada.

6. Resolver dudas y transmitir confianza.

Como podemos apreciar en ambas corrientes el primer objetivo es la consolidación de la relación pediatra-familiares, esto cobra gran importancia ya que se ha identificado que estrategias de soporte no presenciales, como videos educativos, no son suficientes para lograr el objetivo de adherencia a la lactancia materna exclusiva (8), por ejemplo.

\section{¿Cuándo debe realizarse?}

Se recomienda que los obstetras estén prestos a las dudas y preocupaciones que respecto al futuro de su hijo presentan los padres, ante esto deben sugerir la consulta pediátrica prenatal como la fuente de información y educación más apropiada para ellos (4).
Existe cierto grado de consenso respecto a cuándo debe llevarse a cabo esta consulta. En general, la recomiendan durante el tercer trimestre de embarazo, entre las 32 y las 36 semanas de gestación. Se ha descrito que cobra gran importancia en situaciones especiales tales como: primeros embarazos, padres que son nuevos en la práctica, padres solteros, familias con embarazos de alto riesgo, complicaciones del embarazo, gestaciones múltiples, padres que previamente han experimentado una muerte perinatal, así como para los padres que están planeando adoptar un niño (5, 9).

En este punto merecen mención especial los resultados de Goyal et al. quienes mediante una cohorte retrospectiva de mujeres en su primer embarazo, que fueron incluidas en un programa de consulta domiciliaria prenatal antes de las 26 semanas de gestación, identificaron un efecto dosisdependiente del número de visitas para protección de parto pretérmino y tener un recién nacido pequeño para edad gestacional (10).

\section{Beneficios}

La atención pediátrica prenatal ha mostrado ser eficaz para mejorar la salud tanto de la madre como del niño (1). Los beneficios de esta modalidad de atención se aprecian en distintos aspectos: la decisión de lactancia se incrementa, disminuyen las visitas a los servicios de urgencias pediátricas cuando ya ha nacido el niño, ayuda a establecer una relación padres-pediatra más fluida y mejora el cumplimiento en las primeras citas con el pediatra $(1,11)$.

De manera anecdótica, en instituciones colombianas que han implementado la consulta pediátrica prenatal se ha observado una importante disminución en el número de consultas por urgencias en el primer año de vida; sin embargo, esto no ha sido evaluado sistemáticamente.

Quizás el beneficio más estudiado ha sido la decisión de lactancia materna exclusiva. Se ha descrito que la barreras de comunicación evidenciadas por la 
madres durante el asesoramiento prenatal son principalmente respecto a la duración de la lactancia y los métodos para continuar la misma una vez la madre ha retornado al trabajo (12). En el desarrollo de estas estrategias, recientemente Kellams et al. describieron que una estrategia educativa basada en videos fue insuficiente para mejorar la adherencia a las prácticas de lactancia materna (8). Bonuck et al. describieron que su estrategia estructurada con dos consultas prenatales, una posparto y soporte telefónico o domiciliario, aumentaba la intensidad de lactancia (13). Friedman et al. identificaron que la consulta prenatal con un neonatólogo en casos de parto pretérmino influyó positivamente en la duración de la lactancia materna, tanto durante la hospitalización, como en el hogar (14).

Otro de los beneficios estudiados ha sido el de la aceptación de las vacunas. Se ha descrito que las consultas pediátricas prenatales representan el escenario propicio para la educación sobre inmunizaciones(15); sin embargo, Vannice et al. no evidenciaron diferencias en la respuesta positiva materna a la aplicación de vacunas a sus hijos entre madres que recibieron educación prenatal, en comparación con aquellas que recibieron la información en su primera cita para vacunas; aunque se debe resaltar que todas las madres participantes consideran que es preferible recibir la información más tempranamente(16).

Como ya habíamos mencionado, en la cohorte retrospectiva evaluada por Goyal et al., se evidenció que la consulta domiciliaria prenatal fue un factor protector para resultados adversos del embarazo: haber recibido más de ocho visitas prenatales fue un factor protector para parto pretérmino (OR 0,38 95\% IC $0,16-0,87)$, y recibir más de doce lo fue para "pequeño para edad gestacional" (OR 0,32 95\% IC $0.15-0.68)(10)$.

\section{Prácticas recomendadas}

La Academia Americana de Pediatría, realiza las siguientes recomendaciones a los pediatras, referentes a la consulta prenatal (5):
1. Se exhorta a los pediatras a establecer una política para las consultas prenatales. Los servicios ofrecidos pueden ser flexibles y deben estar diseñados para satisfacer las necesidades de los futuros padres. En muchos casos, una visita prenatal completa es ideal, para otros un encuentro breve es suficiente.

2. Comunicación de la política relativa a los pagos por consultas prenatales a los pagadores y las familias. Las sociedades regionales de pediatría deben defender ante las aseguradoras, los beneficios a corto y a largo plazo de las consultas prenatales para la salud de los niños y sus padres.

3. Los pediatras deben compartir sus políticas establecidas para las visitas prenatales con los obstetras locales y con los futuros padres.

4. Durante su formación, los residentes de pediatría debe aprender sobre el contenido y la importancia de las consultas prenatales.

Dejando de lado las sugerencias de la Academia Americana, es importante tener presente que, en algunas circunstancias, como en casos de: padres adolescentes, madres solteras, familiares con enfermedades crónicas, diagnóstico prenatal de defectos congénitos y cuando se espere un parto pretérmino, el apoyo emocional de parte del pediatra hacia los futuros padres debe ser más explícito (4). De otro lado, recordando que dentro de los objetivos de la consulta pediátrica prenatal se encuentra la orientación y educación sobre habilidades de crianza, es importante que el pediatra eduque sobre: alimentación, baño, cambio de pañales, cuidados nocturnos, posición al dormir, uso de chupón, aseo de manos, higiene en casa y otros aspectos clave de los cuidados del recién nacido que son de responsabilidad compartida entre los padres (4).

\section{Perspectivas futuras}

En nuestro país no existen datos publicados referentes a la atención pediátrica prenatal, se sabe que esta es llevada a cabo principalmente en la práctica pediátrica particular y en algunos servicios de medicina prepagada, pero no se conoce o no se ha 
desarrollado un conjunto de recomendaciones para llevarla a cabo por parte de la Sociedad Colombiana de Pediatría.

Realizando una búsqueda en Pubmed con las frases: "Internet impact on pediatric prenatal visit" o "web consultation impact on prenatal visit"; no se logran resultados. Lo que hace pensar que a la fecha no se ha estudiado la influencia de las consultas en la web sobre la actitud de los padres respecto a la atención prenatal. Este sería un tema de gran importancia a evaluar dado que es esta la principal fuente empleada por padres como primera línea de orientación respecto a la salud de los hijos y la propia.

CONFLICTOS DE INTERESES: Los autores no declaran conflictos de interés.

\section{REFERENCIAS BIBLIOGRÁFICAS}

1. Pallás Alonso CR, Adolescencia GPPIy. Prevención prenatal. Visita prenatal. Rev Pediatr Aten Primaria. 2005;7(25):101-6.

2. Merón de Cote PM. Consulta prenatal y seguimiento del recién nacido normal. Pediatr Integral 2014;18(6):384-94.

3. The prenatal visit. American Academy of Pediatrics Committee on Psychosocial Aspects of Child and Family Health. Pediatrics. 1996;97(1):141-2.

4. López-Candiani C. La consulta pediátrica prenatal. Acta Pediátr Mex. 2014;35(1):69-73.

5. Cohen GJ, Committee on Psychosocial Aspects of C, Family H. The prenatal visit. Pediatrics. 2009;124(4):1227-32.

6. Moniz MH, O'Connell LK, Kauffman AD, Singer DC, Clark SJ, Davis MM. Perinatal Preparation for Effective Parenting Behaviors: A Nationally Representative Survey of Patient Attitudes and Preferences. Maternal and child health journal. 2016;20(2):298-305.

7. Hagan JF, Jr., Coleman WL, Foy JM, Goldson E, Navarro A, Tanner JL, et al. The prenatal visit. Pediatrics. 2001;107(6):1456-8.

8. Kellams AL, Gurka KK, Hornsby PP, Drake E, Riffon M, Gellerson D, et al. The Impact of a Prenatal Education Video on Rates of Breastfeeding Initiation and Exclusivity during the Newborn Hospital Stay in a Low-income Population.
Journal of human lactation : official journal of International Lactation Consultant Association. 2016;32(1):152-9.

9. Deborah E. Campbell M. Prenatal Pediatric Visit. In: Thomas K. Mclnerny M, FAAP, editor. American Academy of Pediatrics Textbook of Pediatric Care. 2nd Edition ed: American Academy of Pediatrics; 2016.

10. Goyal NK, Hall ES, Meinzen-Derr JK, Kahn RS, Short JA, Van Ginkel JB, et al. Dosage effect of prenatal home visiting on pregnancy outcomes in at-risk, first-time mothers. Pediatrics. 2013;132 Suppl 2:S118-25.

11. Serwint JR, Wilson ME, Vogelhut JW, Repke JT, Seidel HM. A randomized controlled trial of prenatal pediatric visits for urban, low-income families. Pediatrics. 1996;98(6 Pt 1):1069-75.

12. Taveras EM, Li R, Grummer-Strawn L, Richardson M, Marshall R, Rego VH, et al. Mothers' and clinicians' perspectives on breastfeeding counseling during routine preventive visits. Pediatrics. 2004;113(5):e405-11.

13. Bonuck KA, Trombley M, Freeman K, McKee D. Randomized, controlled trial of a prenatal and postnatal lactation consultant intervention on duration and intensity of breastfeeding up to 12 months. Pediatrics. 2005;116(6):1413-26.

14. Friedman S, Flidel-Rimon O, Lavie E, Shinwell ES. The effect of prenatal consultation with a neonatologist on human milk feeding in preterm infants. Acta paediatrica. 2004;93(6):775-8.

15. Navar AM, Halsey NA, Carter TC, Montgomery MP, Salmon DA. Prenatal immunization education the pediatric prenatal visit and routine obstetric care. American journal of preventive medicine. 2007;33(3):211-3.

16. Vannice KS, Salmon DA, Shui I, Omer SB, Kissner J, Edwards KM, et al. Attitudes and beliefs of parents concerned about vaccines: impact of timing of immunization information. Pediatrics. 2011;127 Suppl 1:S120-6. 